\title{
EL PENSAMIENTO DE MILTON FRIEDMAN EN EL MARCO DE LA ESCUELA DE CHICAGO
}

\author{
Adrián Ravier* \\ enviado: julio 2015 - aceptado: marzo 2016
}

\section{INTRODUCCIÓN}

Milton Friedman, reconocido hoy como uno de los economistas más importantes del siglo $\mathrm{XX}^{1}$, revolucionó a la Escuela de Chicago desde adentro y con ello transformó la ciencia económica, su metodología, la macroeconomía y la política económica.

A un siglo de su nacimiento, este estudio intentará ofrecer un contexto que permita al lector acercarse al estudio de la obra completa de Milton Friedman. Para ello, 1) se identificará el trabajo académico del autor con lo que calificamos como la "segunda etapa" de la Escuela de Chicago; 2) se recorrerá su vida y sus contribuciones; 3) se resumirán las diez proposiciones que sintetizan su pensamiento; 4) se analizarán las políticas económicas que se desprenden de su enfoque; 5) también atenderemos a su lucha contra la inflación tanto teórica como empírica, y se considerará el impacto que estas ideas tuvieron en algunos países; 6) finalmente, se sintetizarán las críticas que Friedman recibió de sus propios colegas libertarios, e intentaremos ofrecer una respuesta para comprender las diferencias y acercar posiciones.

* Es profesor de Economía en la Facultad de Ciencias Económicas y Jurídicas de la Universidad Nacional de La Pampa y también en la Escuela de Negocios de la Universidad Francisco Marroquín, en Guatemala.

1 Cuatro economistas escribieron un artículo en donde sintetizaron los resultados de una encuesta a 299 profesores de economía de Estados Unidos incluyendo preguntas sobre pensadores económicos, revistas científicas y blogs económicos. Para ponderar el orden de los seleccionados, se otorgó 6 puntos al primero, 5 puntos al segundo y 4 puntos al tercero. Ante la pregunta: ¿Quién es su economista favorito del Siglo XX? La respuesta fue John Maynard Keynes, Milton Friedman, Paul Samuelson y Friedrich Hayek, en ese orden. Véase Davis, Figgins, Hedengren, Klein (2011). 


\section{LA ESCUELA DE CHICAGO}

No podemos ignorar a la Escuela de Chicago cuando nos referimos a Milton Friedman, aun cuando este prefería ignorar a las escuelas de pensamiento económico, y en su lugar, concentrar la atención hacia la distinción entre "buena economía" y "mala economía".

La Escuela de Chicago o el Departamento de Economía de la Universidad de Chicago reciben habitualmente una clasificación de tres etapas bien marcadas. La primera comprende desde la creación de la universidad en 1892 — por una donación de John Davison Rockefeller - hasta la segunda mitad de la década de 1940. La segunda comprende desde entonces hasta 1976. La tercera desde allí en adelante.

Diremos que Friedman se formó en la primera etapa; fue el actor principal de la segunda; y que sus ideas y contribuciones tienden a ser abandonadas en la tercera.

En la mencionada primera etapa destacan los padres fundadores del departamento de economía como James L. Laughlin, Thorstein Veblen, John M. Clark, Leon C. Marshall, Frank H. Knight y Aaron Director. ${ }^{2}$ También integraron la primera etapa Jacob Viner, Henry Simons, Henry Schultz, Lloyd Mints y Oskar R. Lange. ${ }^{3}$

Esta variedad de autores denota una heterogeneidad de pensamiento en la primera etapa de la escuela, lo que en efecto explica por qué no se identificó al departamento públicamente como "La Escuela de Chicago" hasta la década de 1930, o incluso hasta 1948, según relata Don Patinkin, fecha en la que él mismo abandona la universidad. ${ }^{4}$

Sin embargo, a partir de la década de 1930 ocurre una serie de cambios fundamentales, destacándose el fallecimiento de Henry Schultz (1938), el abandono de la universidad por parte de Oskar Lange (1945) y Jacob Viner (1946) y las incorporaciones de Milton Friedman (1946) y Lloyd A. Metzler (1947). George J. Stigler recién se suma en 1958.

La transformación de la heterogénea Escuela de Chicago de la primera etapa a una más homogénea, hoy identificada en gran parte con el pensamiento de Friedman, se da a través de Frank Knight y sus alumnos, especialmente el propio

Véase Van Overtveldt (2007): The Chicago school, Agate.

Véase Rutherford (2010).

4 Véase Patinkin (1969). 
Friedman, Stigler y Allen Wallis. Juan Carlos de Pablo nos comenta que los alumnos de Jacob Viner no fueron un club y si bien se esperaba mucho de Oskar Lange, este retornó a Polonia para desarrollar una carrera política. ${ }^{5}$

Las más conocidas contribuciones de esta segunda etapa surgen en torno al campo monetario - sobre las que volveremos más adelante-, pero también hay que destacar otras áreas como la economía agrícola (Theodore W. Schultz), capital humano (Jacob Miner, T. W. Schultz y Gary Becker), economía laboral (Harold G. Lewis, Albert E. Rees, Sherwin Rosen, James J. Heckman, Edward Lazear), mercados financieros eficientes (Eugene F. Fama, Fisher Black y Myron S. Scholes), regulación y desregulación (George J. Borjas, Harold Demsetz, Bruce L. Gardner, Elizabeth M. Landes, Peter Linneman, Bedros P. Pashigian, Sam Peltzman, Richard A. Posner y George Stigler), derecho y economía (Aaraon Director, Edward H. Levi, Ronald Coase, R. A. Posner, Armen A. Alchian y H. Demsetz), enfoque monetario de la balanza de pagos (Harry G. Johnson y Robert A. Mundell), historia económica (Earl Hamilton, Robert Mc Closkey y David Galenson) y estadística y econometría (Lars P. Hansen, Arnold Z. Zvi Griliches y James Heckman). ${ }^{6}$

A partir de 1976, surge una tercera etapa en Chicago, donde se visualizan dos grupos. El primero, liderado por Gary Becker, quien utiliza las herramientas del análisis económico para estudiar temas variados como la discriminación, el crimen, el capital humano, las familias, el mercado de órganos y la política, por solo mencionar algunos. El segundo, liderado por Robert Lucas, se construye sobre las expectativas racionales de John F. Muth ${ }^{7}$ en un contexto microeconómico y desde allí transforma la macroeconomía y la política económica, creando lo que hoy se conoce como la Escuela de Expectativas Racionales o la Nueva Macroeconomía Clásica. ${ }^{8}$

En definitiva, como ha señalado Larry A. Sjaastad, profesor de Chicago durante más de 40 años, la "Escuela de Chicago de Friedman-Stigler-Harberger" lamentablemente dejó de existir, o como dirían las nuevas generaciones de profesores "volvió a transformarse". 9

\footnotetext{
5 Véase De Pablo (2011).

6 Véase De Pablo (2011), op. cit.

7 Véase Muth (1961).

8 Véase Rosende (1995).

9 Véase Sjaastad (2011).
} 


\section{MILTON FRIEDMAN (1912-2006)}

Milton Friedman se ha definido a sí mismo como un hombre de suerte. ${ }^{10}$ Él mismo señala que el primer hecho fortuito surge con su nacimiento, gracias a que sus padres emigraron a tiempo a los Estados Unidos para que pudiera ser un ciudadano americano con plenos derechos.

Hijo de inmigrantes judíos, Milton Friedman nació en Brooklyn, Nueva York, el 31 de julio de 1912. Poco después de su nacimiento, su familia se trasladó a Nueva Jersey, donde creció y comenzó a mostrar su talento en la academia al completar sus estudios en la Escuela Superior de Rahway, antes de sus 16 años. Allí emergió el segundo hecho fortuito, cuando un profesor de geometría le descubrió el paralelo entre la Ode on a Grecian Urn — el famoso poema de John Keats - y el teorema de Pitágoras, y así pudo apreciar la belleza matemática.

La suerte siguió de su lado. En el contexto de la Gran Depresión obtuvo una beca para estudiar en la Universidad de Rutgers, donde se especializó en matemáticas, y hasta pensó en convertirse en actuario. Friedman mismo destaca un cuarto hecho fortuito, ya que en Rutgers conoció a dos profesores que resultaron ser sumamente inspiradores, Homer Jones y Arthur F. Burns, quienes lo convencieron de que la economía podía ayudar a poner fin a la Gran Depresión. Graduado en 1932, fue el propio Homer Jones quien intervino personalmente para que le sea concedida una beca de posgrado en la Universidad de Chicago (dejando a un lado otra beca igualmente atractiva — para estudiar matemática aplicada en la Brown University). ${ }^{11}$

Friedman destaca que fue un quinto hecho fortuito el haberse sentado, por orden alfabético, al lado de Rose Director, quien más tarde fuera su esposa y compañera. Teniendo como compañero a George Stigler, Friedman completa su maestría en 1933, recibiendo las enseñanzas de Frank Knight, Lloyd Mints, Henry Simons, Henry Schultz y Jacob Viner, siendo el curso de este último "incuestionablemente la mayor experiencia intelectual de mi vida".

10 Véase Friedman (1986) \& Ávila (2006).

11 "It was close to a toss of a coin that determined which offer I accepted". Véase Friedman (2004): "In Lives of the Laureates", 4th ed., edited by William Breit and Barry T. Hirsch, 65-77. Cambridge, Mass.: MIT Press, p. 69. Citado por Cole (2007): "Milton Friedman 1912-2006”, The Independent Review: A Journal of Political Economy, Summer 2007, XII (1), 115-128. 
Durante el período 1933-1934 Friedman recibe otra beca para estudiar en la Universidad de Columbia, donde estudia estadística junto a Harold Hotelling, un influyente economista teórico y reconocido estadístico-matemático.

En 1934-1935 retorna a la Universidad de Chicago como asistente de Henry Schultz, quien entonces trabajaba en la teoría de la demanda y su posible medición. En aquel año, Friedman se reencuentra con George Stigler, quien se convierte en amigo y compañero de trabajo por el resto de sus días.

Ya en 1935 y con ciertas dificultades para encontrar empleo, Friedman se dirige a Washington, donde el New Deal de Roosevelt había creado ciertos empleos para salvar a varios jóvenes economistas. Ese mismo año trabaja en el Comité de Recursos Naturales, donde se desarrolla una gran encuesta sobre consumo, con enorme presupuesto. Friedman toma entonces algunas ideas que más tarde se vuelven centrales en su trabajo sobre una teoría de la función de consumo.

Durante el otoño de 1937 Friedman llega al National Bureau of Economic Research (NBER), donde asiste a Simon Kuznets en un estudio sobre ingresos, el que termina siendo un trabajo en coautoría, en el que por primera vez distingue entre ingreso transitorio e ingreso permanente. ${ }^{12}$

En 1940 Friedman obtiene una posición como docente en la Universidad de Wisconsin-Madison, pero el antisemitismo en el Departamento de Economía, lo lleva a abandonar el cargo y retornar a los servicios del gobierno.

Durante la Segunda Guerra Mundial, y en particular entre 1941 y 1943, Friedman trabajó en política fiscal para el gobierno federal, como asesor de altos funcionarios del Departamento de Economía y Hacienda. Muchos aún critican sus políticas keynesianas sobre los impuestos, a las que él mismo reconoce más tarde como un paso en falso.

En 1943 Friedman regresa a la Universidad de Columbia, y con ello reinicia una vida académica que ya no abandonará. Al principio se une a una división de investigaciones sobre guerra, donde realiza estudios estadísticos, focalizándose en problemas sobre diseño de armas, tácticas militares y experimentos metalúrgicos. Luego, ya en 1945, presenta aquel trabajo en coautoría con Kuznets sobre ingresos como su tesis doctoral, la que le permite alcanzar el doctorado en economía

12 Véase Friedman \& Kuznets (1937, 1938, 1939), M. Friedman \& S. Kuznets (1939). 
en 1946. ${ }^{13}$ Mientras tanto, entre 1945-1946, retoma la docencia en la Universidad de Minnesota, donde George Stigler también ocupaba un cargo. Y también en 1945 nace su hijo, David Friedman.

En 1946 Friedman acepta un ofrecimiento como profesor de teoría económica en la Universidad de Chicago, oportunidad abierta gracias a su antiguo profesor, Jacob Viner, quien se traslada a la Universidad de Princeton. Desde entonces, Friedman trabajará en la Universidad de Chicago por treinta años, transformando las ideas y construyendo una comunidad intelectual que ya ha producido 12 premios Nobel en economía, si consideramos solo a sus profesores, o 22 si incluimos también a los estudiantes.

Casi simultáneamente con su retorno a Chicago, Arthur Burns, entonces director del NBER, consulta a Friedman si desea conformar el staff del Bureau. Friedman acepta la invitación, y asume la responsabilidad para investigar el rol de la política monetaria en los ciclos económicos. Como resultado, inicia el "Chicago Workshop", un taller sobre moneda y banca que promueve un "revival" sobre estudios monetarios y un renovado interés por la teoría cuantitativa del dinero. Durante la segunda mitad de la década de 1940 Friedman colabora con Anna Schwartz, una historiadora del Bureau, que resultará en la publicación del libro en coautoría titulado A Monetary History of the United States, 1867-1960, publicado en 1963.

Friedman destina los años 1954 y 1955 a una beca Fulbright en Gonville and Caius College, en la Universidad de Cambridge. Por esos tiempos, los economistas de Cambridge estaban divididos entre una mayoría keynesiana, que incluía a Joan Robinson y Richard Kahn y una minoría antikeynesiana, liderada por Dennis Robertson. Friedman especula que fue invitado a participar porque su visión era contraria a ambas posiciones.

Durante 1964, sus consejos económicos llegaron al candidato a presidente republicano Barry Goldwater. Más tarde, entre 1966 y 1984, sus columnas semanales en la revista Newsweek fueron cada vez más leídas y su influencia creciente llegó a la política y a los hombres de negocio.

En 1976 Friedman recibe el Premio Nobel en Economía, "por sus contribuciones en los campos del análisis del consumo, la historia y la teoría monetaria y por su demostración de la complejidad que significa la política de estabilización".

13 Véase Friedman \& Kuznets (1945). 
Durante 1977, a los 65 años de edad, Friedman se retira de la Universidad de Chicago, después de enseñar durante treinta años. Junto a su esposa se traslada a San Francisco. Desde 1977 se afilia al Hoover Institution de la Stanford University. Durante ese mismo año, Friedman se ocupa del proyecto "Libertad de Elegir" y solicita crear un programa de televisión donde presentar su filosofía económica y social. Milton y Rose Friedman trabajan en este proyecto por los siguientes tres años, y durante 1980, la serie de diez partes, titulada "Libertad de Elegir", sale al aire a través de la televisión pública. Luego de la serie, Friedman, en coautoría con su esposa, Rose Friedman, publica el libro, que lleva el mismo nombre, el cual se convierte en best seller de 1980 y se traduce a catorce idiomas.

En 1980 Friedman sirve como un consejero no-oficial durante la campaña presidencial de Ronald Reagan, y luego se convierte en Presidente del Consejo de Política Económica por el resto de la administración Reagan. En 1988, Friedman recibe la National Medal of Science y Reagan lo honra con la Medalla Presidencial de la Libertad. Durante las décadas de 1980 y 1990, Friedman continúa escribiendo columnas y aparece en televisión. También viaja a Chile, el Este de Europa y China, para aconsejar a algunos gobiernos.

Milton Friedman fallece de un ataque al corazón el 16 de noviembre de 2006 en un hospital de San Francisco.

\section{II.1. La economía positiva y el monismo metodológico}

La primera contribución de Milton Friedman se encuentra en sus Essays on Positive Economics, y en particular en su "Metodología de la Economía Positiva", donde argumenta que la utilidad de una teoría, en las ciencias naturales y en las ciencias sociales, depende del éxito de sus pronósticos y no del realismo descriptivo de sus supuestos. ${ }^{14}$ No solo ello, Friedman además enfatiza el "no realismo", señalando que "cuanto más significativa sea la teoría, más irreales serán sus supuestos".

14 El artículo original en inglés llevaba como título The Methodology of Positive Economics; la versión castellana puede verse en el libro Ensayos sobre economía positiva, Gredos, Madrid, 1967, p. 9. La bibliografía secundaria «clásica» puede verse en estos papers: Nagel, E. (1963); Musgrave, A., (1981); Boland, L (1979) y Caldwell, B.,(1980); todos reimpresos en Caldwell, B. (1984). Dentro de la inagotable bibliografía posterior, véanse los artículos de Mayer, Maki, Hands, Boland y Reder en el n. ${ }^{\circ} 4$ vol. 10 de The Journal of Economic Methodology, December 2003. 
Una lectura habitual de esta última cita es que Friedman es en parte responsable del abuso de la matemática en economía. Así, es costumbre en nuestros días el crear modelos bajo ciertos supuestos que determinan el resultado que el economista quiere alcanzar, lo cual lleva a los economistas a caer en el cientismo, en lugar de construir ciencia.

Sin embargo, existe otra lectura de esta contribución de Friedman que merece mayor atención, y que ha sido señalado por los citados Nagel, Musgrave y Caldwell. Como bien resume el profesor Zanotti, Friedman no estaba afirmando un tosco "no realismo" de las hipótesis, sino un "no completo realismo" de estas. Toda hipótesis, en el método hipotético-deductivo, no es una copia de la realidad (el mapa no es el territorio), sino una simplificación predictivamente relevante (y por eso Friedman las llamó "descriptivamente falsas"). Visto así, lo más discutible de su ensayo no es esa "obviedad" (a saber: que un completo realismo es imposible), sino la relación inversa que plantea entre ese no completo realismo (obvio) de las hipótesis y su poder predictivo, y ese es el punto que destaca Musgrave. ${ }^{15}$

Friedman no volvió sobre el campo metodológico en muchas ocasiones, pero sí lo hizo en su conferencia de 1976, en Estocolmo. ${ }^{16}$ De hecho, allí Friedman fue uno de los primeros en destacar el escepticismo generalizado que existía en torno a la economía como ciencia, cuando se estableció el premio Nobel a una disciplina que muchos pensaban diferente de otras "ciencias exactas", como la física, la química y la medicina, en las que sería posible un conocimiento objetivo, acumulativo, definitivo.

Después de que Karl Popper demostrara que en "ninguna" ciencia, y tampoco en las mencionadas "exactas", es posible confirmar definitivamente una hipótesis, Friedman hace bien en señalar la subestimación que se hace de la economía, pero también la sobreestimación que reciben otras ciencias. ${ }^{17}$

15 Véase Zanotti (2011).

16 Véase Friedman (1976).

17 Véase Popper (1934). En este sentido, Friedman destaca un monismo metodológico con el que coincidimos. Como bien señala Zanotti (1996): "Después de Popper, no se puede sostener que las hipótesis de las ciencias naturales puedan testearse con toda certeza". Pero en otro sentido, preferimos hablar de un dualismo metodológico incompatible con el homo economicus del análisis neoclásico que Friedman apoya. Y es que los agentes en la física carecen de la "acción humana" a la que se enfrenta el analista económico. En física los agentes reaccionan. Ante un experimento se comportan siempre del mismo modo. En economía, la acción humana varía de un sujeto a otro y de un momento a otro. Cada individuo es único. Es por ello que el individualismo metodológico y el subjetivismo metodológico resultan elementos fundamentales en el análisis económico. El agente 
Ya en 1953 Friedman se presenta como un defensor del "monismo metodológico" y también como un "positivista", en el sentido de que toda ciencia para ser tal debe probar sus hipótesis a través del método científico, esto es, con hechos reales contrastados por la experiencia.

Aclara por supuesto que en la economía no es posible realizar "experimentos controlados", diseñados explícitamente para estudiar una hipótesis y aislar las variables bajo estudio de otras fuerzas perturbadoras. En su lugar, propone apoyarse sobre "experimentos que ocurren casualmente", algo que desde su punto de vista solo distingue a la economía de la física en una cuestión de grado.

El experimento "crucial" no es posible en economía. El proceso de descarte de hipótesis falsas es más lento que en otras ciencias. Pero en ocasiones la experiencia proporciona evidencia lo suficientemente contundente como para que se asemeje a los resultados de un experimento controlado. Tal es el caso de la relación empírica entre el crecimiento monetario y la inflación de precios, para citar un ejemplo. ${ }^{18}$

\section{II.2. La teoría de la función consumo}

El segundo aporte de Friedman es lo que él mismo define como su mejor trabajo científico, esto es, su teoría de la función consumo de 1957, donde justamente aplica su aporte metodológico previo. ${ }^{19}$

Allí sostiene que el nivel de consumo de los agentes no depende de ingresos transitorios, sino del ingreso permanente, el esperado de largo plazo. Esto tiene dos implicaciones: por un lado, no hay motivo para que el capitalismo sufra un estancamiento por subconsumo, como señaló Keynes en su Teoría General de la Ocupación, el Interés y el Dinero (1936); por otro lado, puso en duda la política fiscal contracíclica, la que no debería tener un impacto apreciable sobre la actividad económica puesto que los consumidores ignorarán los cambios transitorios en el ingreso disponible asociados a rebajas o aumentos de tributos.

\section{II.3. Evidencia empírica para la teoría cuantitativa del dinero de Irving Fisher}

\footnotetext{
representativo no puede incorporar al análisis dicha subjetividad. Para profundizar en este punto se recomienda el libro de Gerald P. O’Driscoll y Mario J. Rizzo (2010). Véase también Zanotti (2007).

18 Véase Cole (1991).

19 Véase Friedman (1972). Versión en español por Lorenzo Betancor C., Alianza Editorial, Madrid, 1972.
} 
La tercera contribución científica de Friedman está relacionada con la teoría monetaria. Consiste en haber recuperado el interés de los economistas por la teoría cuantitativa del dinero, la que fuera enunciada original y rudimentariamente por Jean Bodin ${ }^{20}$ y los teóricos de Salamanca en el siglo XVI, ${ }^{21}$ retomada más tarde por John Locke, David Hume 22 y varios de los clásicos, formulada en una ecuación consistente por Irving Fisher en 1911, ${ }^{23}$ pero popularizada recién con los escritos empíricos de Milton Friedman.

$\mathrm{Al}$ respecto, cabe señalar la conexión, a veces ignorada, entre Irving Fisher y Milton Friedman, en particular en economía monetaria, comprendiendo la teoría cuantitativa del dinero, el efecto Fisher, las expectativas inflacionarias, la paradoja de Gibson, la teoría monetaria de los ciclos económicos, la curva de Phillips y hasta los rezagos empíricos de la política monetaria. Michael D. Bordo y Hugh Rockoff también extienden la influencia hacia la política monetaria, en particular sobre algunos esquemas de reforma monetaria tratados por Friedman, como la regla de aumentar la cantidad de dinero un $\mathrm{k} \%$, el congelar la base monetaria, el dólar compensado, el mandato para la estabilidad de precios, el 100\% como coeficiente de reserva y hasta el dinero sellado. ${ }^{24}$

La influencia se dio tanto directa como indirectamente. Por un lado, Friedman consideraba a Fisher el economista americano más importante que haya dado la historia, cuya obra estudió en profundidad e incluyó extensas referencias en su trabajo. Por otro lado, la influencia va de Fisher hacia la Escuela de Chicago de la década de 1930 — en particular, sobre el pensamiento de Frank Knight y Henry Simons-, y de allí al pensamiento de Friedman. ${ }^{25}$

Pero lo cierto es que la Escuela de Chicago de la segunda etapa se identifica fuertemente con la teoría cuantitativa del dinero, la que señala una relación directa entre la cantidad de dinero por un lado y los precios por el otro. Es cierto que esta noción es una de las más viejas de la economía, pero una cosa es expresar la idea en términos generales y otra cosa es sistematizar la relación entre el dinero por un lado y los precios y otras magnitudes por el otro.

20 Véase Bodin (1569).

21 Véase Huerta de Soto (2002), cap. 2 y 9.

22 Véase Hume (1970 [1752]).

23 Véase Fisher (1963 [1911]).

24 Véase Bordo y Rockoff (2011).

25 Véase Friedman, Jones, Stigler, y Wallis (Comp.) (1935): "Frank H. Knight”, The Ethics of Competition, George Allen \& Unwin, Londres, 1935. Véase también Friedman (1967). 
Esta ecuación cuantitativa — que debemos a Fisher- se resume en la siguiente expresión:

$$
\mathrm{MV}=\mathrm{Py}
$$

lo que significa que la masa monetaria (M) multiplicada por la velocidad de circulación (V) es igual al nivel general de precios, multiplicado por el volumen de transacciones (y). En pocas palabras, Fisher interpretó que la velocidad de circulación podía considerarse altamente estable, que podía tomarse como determinada en forma independiente de los otros términos de la ecuación, y que como resultado de esto los cambios en la cantidad de dinero se reflejarían en los precios o en la producción.

Para ser más preciso, Fisher explicaba que la política monetaria altera la estructura de precios relativos, con ello el patrón de la producción real, y solo en el largo plazo el nivel general de precios. Esta noción se vuelve más tarde en una de las proposiciones centrales de la Escuela de Chicago de la segunda etapa.

\section{II.4. La historia monetaria de los Estados Unidos}

Volviendo sobre Friedman, su interés por el campo monetario nace prácticamente con su incorporación a Chicago y al NBER en 1946. El mencionado "Chicago Workshop" sobre moneda y banca es justamente el que origina una serie de trabajos empíricos conocidos bajo el título Studies in the Quantity Theory of Money, que además dan nacimiento a la segunda etapa de la Escuela de Chicago. ${ }^{26}$

Por el lado de sus investigaciones en NBER, Friedman retoma junto a Anna J. Schwartz un proyecto ambicioso de Wesley Mitchell sobre el impacto de la política monetaria en los ciclos económicos, trabajo que origina su cuarta contribución a la ciencia económica, o más bien a la historia económica. ${ }^{27}$

Tal es así que en A Monetary History of the United States, 18671960, Friedman y Schwartz demuestran el rol activo que tuvo la política monetaria sobre la actividad económica, señalando que cada ciclo económico que experimentó el país encuentra su causa en la inestabilidad de la política monetaria. En particular, en la Gran Depresión, se atribuyen errores al directorio de la Reserva Federal y

26 Véase Friedman (1956)

27 Véase Friedman (1950). 
no a una estabilidad inherente de la inversión en el sistema capitalista como se postuló en la posguerra. ${ }^{28}$

Pero no se trata únicamente de esta conocida obra magistral, sino también de otro trabajo asociado, escrito por Phillip Cagan, bajo el título Determinants and Effects of Changes in the Stock of Money, 1875-1960, ${ }^{29}$ publicado poco después. Un tercer volumen, también publicado por Friedman y Schwartz fue Monetary Statistics of the United States, publicado en 1970.30 Y finalmente, también por Friedman y Schwartz, Monetary Trends in the United States and the United Kingdom, publicado en 1982. ${ }^{31}$

De esta manera, Friedman y su equipo, popularizan la teoría cuantitativa del dinero en una dimensión empírica hasta ese momento desconocida, colocando cierto énfasis en la demanda de dinero, ${ }^{32}$ además de la oferta monetaria. Por su parte, se retoma el interés sobre la velocidad de circulación del dinero, la que conecta entonces el ingreso con la masa monetaria, y se señala el rezago como un factor determinante. 33

\section{II.5. El rol de la política monetaria}

De más está decir que no estamos cubriendo aquí toda su literatura — sería imposible en el espacio del que aquí disponemos-, pero no podemos dejar de mencionar The Role of Monetary Policy de 1967, mismo título que presentó en su discurso como presidente de la American Economic Association. ${ }^{34}$ Para muchos autores, esta quinta contribución resultó en un aporte aún más importante que sus estudios sobre historia y teoría monetaria, en particular por elaborar una predicción que hizo en la década de 1950, cuando sostenía que había una falla fundamental en la teoría macroeconómica keynesiana: "Esperen que el desempleo aumente y que la inflación suba, al mismo tiempo".

Friedman ejerció una reacción teórica muy profunda sobre la curva de Phillips de pendiente negativa de 1958, que si bien fue ignorada en los primeros años, fue aceptada pocos años más tarde.

28 Véase Friedman y Schwartz (1963a y 1963b).

29 Véase Cagan (1965). El libro cuenta con un prólogo de Friedman.

30 Véase Friedman y Schwartz (1963): Monetary Statistics of the United States, Columbia University Press, New York, 1970.

31 Véase Friedman y Schwartz (1982).

32 Véase Friedman (1959b).

33 Véase Friedman (1961).

34 Véase Friedman (1968). 
Incluso para Paul Krugman —un autor que se encuentra en las antípodaseste fue uno de los logros intelectuales decisivos de la posguerra. Mucho más aún cuando se observó que el modelo de Phillips, si bien se adaptó correctamente en su aplicación al caso de Estados Unidos, ${ }^{35}$ falló en su aplicación a otras economías. Sobrevino entonces el proceso de "estanflación", entendido como un fenómeno de alta inflación y alto desempleo simultáneamente que, como correctamente señala Milton Friedman, "volvió algo ridículos los confiados comentarios de muchos economistas sobre 'trade off' de inflación por desempleo, basados en curvas Phillips empírica a medida." 36

II.6. La contrarrevolución monetarista y la curva de Phillips vertical ajustada por expectativas

Se produjo entonces lo que Milton Friedman (1976) denominó como la "contrarrevolución monetarista", ${ }^{37}$ la que relegó el estudio keynesiano solo en el corto plazo y replanteó una nueva curva de Phillips, ahora de pendiente vertical, dando lugar a un nuevo programa de investigación. ${ }^{38}$ Esta es en efecto, su sexta contribución científica.

Este programa alternativo incluía la teoría cuantitativa del dinero como eje y otorgaba mayor relevancia a la distinción entre el efecto de la política monetaria en el corto y el largo plazo, prestando atención al rezago de la política monetaria y a la neutralidad del dinero en el largo plazo y creando tres nuevas teorías o conceptos: la de

35 Véase Samuelson y Solow (1960).

36 Véase M. Friedman (1992 [1975]), "Unemployment versus inflation", IEA, Lecture n. ${ }^{\circ}$, Occasional paper n. ${ }^{\circ} 44$, Londres. Existe una versión en español bajo el título “¿Desempleo versus inflación? Evaluación de la Curva Phillips", en La economía monetarista, editado por Gedisa, Barcelona, noviembre 1992, p. 89.

37 Véase Milton Friedman (1970). Véase también Johnson (1984).

38 Axel Leijonhufvud (2000, p. 515), en un artículo donde se cuestiona qué han hecho los modernos con Keynes, replantea la historia de la curva de Phillips señalando que "la economía keynesiana floreció durante unos veinticinco años sin que hubiera curvas de Phillips. Este fue un agregado tardío. Por otra parte, el propio Phillips no creía que las regularidades que había descubierto en los datos se mantuvieran en condiciones inflacionarias. La econometría de las curvas de Phillips fue vacilante desde el principio y la 'curva' nunca tuvo un fundamento teórico comprensible. Por todo esto, son muchos los que descreyeron de esa construcción aún antes de la revolución de las expectativas racionales (ver, por ejemplo, Leijonhufvud, 1968)". En el mismo sentido argumenta Gerald O'Driscoll (2009, p. 173), quien prefiere hablar de una contrarrevolución de la "macroeconomía neoclásica", la que "evolucionó en una síntesis de keynesianismo y monetarismo (no necesariamente representada por Keynes y Friedman)". 
las expectativas adaptativas, la tasa natural de desempleo ${ }^{39}$ y la teoría aceleracionista de la inflación. ${ }^{40}$. La curva de Phillips vertical monetarista se expone en la figura 1.

Sin caer en tecnicismos, podemos exponer su idea esencial en forma resumida. Imaginemos una economía con cierto desempleo y estabilidad de precios (punto E). Esto último implica que las expectativas inflacionarias serán consistentes con "inflación cero", dado que la autoridad monetaria no ha practicado en el pasado cercano políticas inflacionarias. Ahora supongamos que la autoridad monetaria decide - bajo la receta keynesiana - expandir el crédito para alcanzar el pleno empleo (punto F). Friedman de alguna manera acepta dicha tesis de que tal política puede ser efectiva en el corto plazo. Pero advierte que tal cosa solo ocurre por un efecto "sorpresa" en los trabajadores que no advierten rápidamente la caída que tal política ejerce sobre sus salarios reales y el poder adquisitivo. Ahí es donde Friedman introduce su concepción de expectativas adaptativas. Una vez que los trabajadores visualizan el efecto reclaman a través de los sindicatos por una recuperación del salario real. Una vez que estos reclamos producen una recuperación en el salario real, el efecto de corto plazo desaparece, se neutraliza, y la economía vuelve al mismo punto de desempleo que teníamos al momento inicial (punto G). Esto es el conocido principio de la "neutralidad del dinero de largo plazo". En definitiva, Friedman explica que en toda economía hay una "tasa de desempleo

39 En este punto hay que destacar también las contribuciones de Edmund Phelps, que le valieron el Premio Nobel de 2006. Véase Phelps (1971); Phelps (1967); Phelps (1968).

40 Esta hipótesis también fue defendida por Friedrich Hayek: "La inflación crea 'temporalmente' algunos puestos de trabajo que, sin embargo, desaparecen cuando se pasa aquélla o cuando disminuye su ritmo de aceleración. Esto es así porque la inflación: a) Cambia la distribución de dinero entre los diversos sectores y etapas del proceso de producción. b) Crea una expectativa de ulteriores subidas de precios. Los defensores de la política monetaria de pleno empleo se imaginan que sería suficiente un único aumento de la demanda total para asegurar el pleno empleo durante un tiempo indefinido, aunque duradero. Tan pronto como el gobierno asume la responsabilidad de mantener el pleno empleo, sean cuales fueren los salarios que los sindicatos consiguen, entonces estos últimos se desentienden del paro que sus demandas salariales puedan producir. En esta situación, cada subida de jornales por encima del aumento de productividad forzará un incremento de la demanda total si se quiere evitar el paro. El aumento de la cantidad de dinero provocado por esta escalada de salarios se convierte en un proceso continuo que exige nuevas y continuas sumas dinerarias adicionales. Esta oferta adicional de dinero conduce a cambios en la demanda relativa de los diversos bienes y servicios, lo que provoca ulteriores trastornos en los precios relativos en el curso de la producción y en la asignación de los factores de producción, entre ellos el trabajo. [...] La conclusión principal a la que puedo llegar es que cuanto más dure la inflación mayor será el número de trabajadores cuyos empleos dependerán de la 'continuación' de la inflación, incluso, muy frecuentemente, de una 'aceleración' continua de la misma, y ello no porque ellos se hubieran quedado cesantes en ausencia de inflación, sino porque fueron atraídos a trabajos que la inflación hizo temporalmente atractivos, pero que desaparecen tan pronto como cesa el ritmo de inflación o desaparece ésta”. Véase Hayek (1975), pp. 48-49. 
Figura 1. La curva de Phillips ajustada

Tasa de Inflación

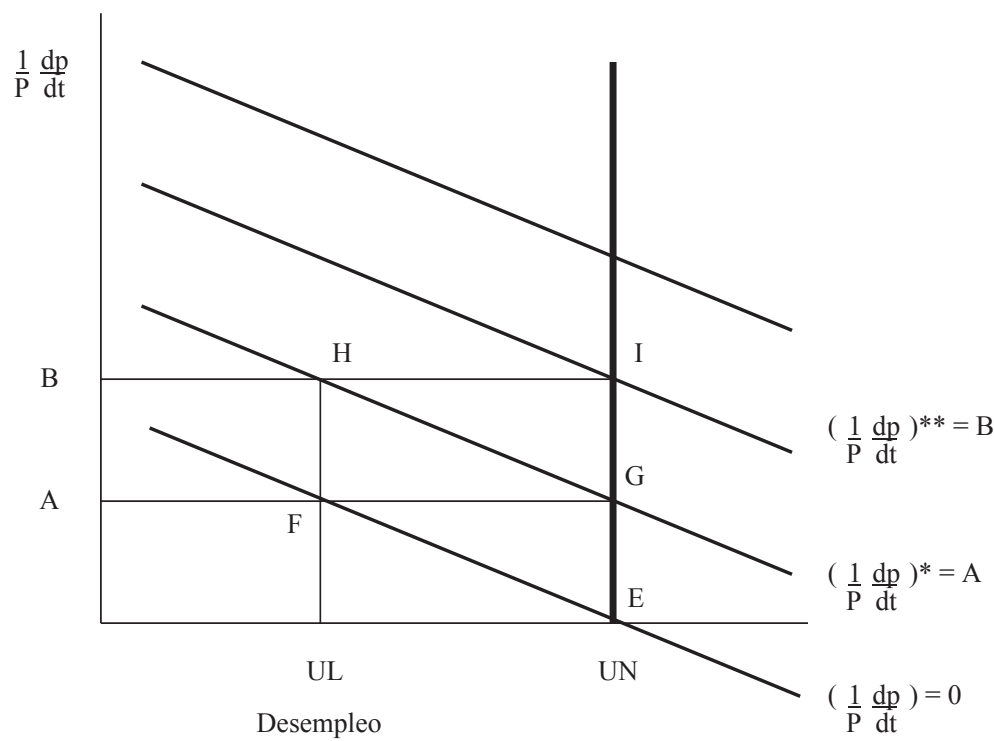

Fuente: elaboración propia en base a Friedman (1970)

natural", que será consistente con la realidad económica subyacente, y con cierta legislación y salario mínimo que prevalecen en la economía. La política monetaria puede permitir un abandono de dicha tasa en el corto plazo, pero en el largo plazo, tales efectos se neutralizan, y la economía retorna a su mismo nivel.

Es cierto, sin embargo, que una política aceleracionista de la inflación, esto es, una política aún más agresiva que lo que los agentes o trabajadores esperan bajo sus expectativas, puede provocar un nuevo efecto sorpresa (punto $\mathrm{H}$ ). Pero más tarde las expectativas nuevamente se adaptarán a la nueva situación, el efecto real se anulará, y lo único que queda es el efecto sobre los precios (punto I).

De aquí es que Friedman concluye que los hacedores de políticas públicas o policymakers debieran abandonar la idea de alcanzar el pleno empleo mediante políticas monetarias expansivas, porque lo único que conseguirán es una aceleración de la inflación, sin lograr un efecto duradero en la generación de empleo. 


\section{II.7. Las diez proposiciones del monetarismo}

A continuación, se resumen las diez proposiciones que Friedman considera centrales en el monetarismo que él mismo contribuyó a construir:

1. Hay una relación coherente, aunque no precisa, entre la tasa de crecimiento de la cantidad de dinero y la tasa de crecimiento del ingreso nominal.

2. Esta relación no se hace evidente a simple vista porque los cambios en el crecimiento monetario tardan en afectar el ingreso.

3. En promedio, un cambio en la tasa de crecimiento monetario produce un cambio en la tasa de crecimiento nominal entre los 6 y 9 meses más tarde. Friedman analizó los casos de Japón, India, Israel y los Estados Unidos. Algunos estudiantes han investigado los casos de Canadá y de una cantidad de países sudamericanos. No importa qué país se estudie, generalmente hay una demora de 6 a 9 meses.

4. Los cambios en la tasa de crecimiento del ingreso nominal típicamente se reflejan antes en la producción y casi nada en los precios.

5. En promedio, el efecto sobre los precios viene entre 6 y 9 meses después del efecto sobre el ingreso y la producción, así que la demora total entre un cambio en el crecimiento monetario y un cambio en la tasa de inflación (entendida como subida sostenida y generalizada de los precios) es en promedio de 12 a 18 meses. De aquí Friedman deduce que la escalada de precios no se puede detener de la noche a la mañana.

6. Incluso tomando en cuenta la demora en el efecto del crecimiento monetario, la relación está lejos de ser perfecta. Los cambios en el corto plazo no son "proporcionales".

7. En el corto plazo, que puede ser cinco o diez años, los cambios monetarios afectan primordialmente la producción. Por el otro lado, midiendo por décadas, la tasa de crecimiento monetario afecta primordialmente a los precios.

8. De las proposiciones que presentamos hasta aquí se deduce que "la inflación es siempre y en todas partes un fenómeno monetario" en el sentido de que es y solo puede ser producida por un aumento más rápido de la cantidad de dinero que de la producción.

9. El gasto gubernamental puede o no ser inflacionario. Lo será en la medida en que sea financiado con la creación de dinero, es decir, imprimiendo moneda o creando depósitos bancarios. De otra forma, no tendrá relación.

10. Los cambios en la cantidad de dinero afectan las tasas de interés en una dirección al principio, pero más tarde en la dirección opuesta. El crecimiento monetario más rápido al principio tiende a bajar las tasas de interés. Pero más tarde, a medida que aumenta el gasto y estimula la subida inflacionaria de precios, también produce un aumento en la demanda de préstamos, lo que tenderá a 
aumentar las tasas de interés. Esa es la razón por la cual a nivel mundial las tasas de interés son más bajas en los países que han tenido la tasa de crecimiento más lenta en la cantidad de dinero — países como Suiza y Alemania—.

\section{II.8. Expectativas racionales y curva de Phillips}

En la actualidad, un doctorando en economía de la Universidad de Chicago ya no se forma bajo estas proposiciones. Y es que hoy la concepción monetarista de la curva de Phillips es rechazada y a la vez reforzada por la Nueva Macroeconomía Clásica, representada por Robert Lucas de la Universidad de Chicago y Thomas Sargent del Instituto Hoover. ${ }^{41}$

Estos autores reemplazaron el concepto de expectativas adaptativas por el de expectativas racionales, lo que los llevó a extender la "neutralidad del dinero" también al corto plazo.

La conclusión de estos autores es que la política monetaria no solo tendrá efectos neutrales en el largo plazo, sino también en el corto plazo. Bajo expectativas racionales, los agentes no pueden ser sorprendidos por la política monetaria. En pocas palabras, la política monetaria ya no creará empleo, ni en el corto, ni en el largo plazo.

\section{II.9. La curva de Phillips de pendiente positiva}

Merece especial atención, sin embargo, lo que Friedman denomina en su primer ensayo de este libro como la tercera etapa del estudio de la curva de Phi1lips. Es lo que yo mismo he llamado "la observación de Friedman", ${ }^{42}$ y lo que podríamos calificar como la séptima contribución científica de este autor.

Así como la evidencia empírica conocida como "estanflación” invalidó el estudio original de Phillips y fortaleció su propia tesis de una curva de Phillips de pendiente vertical, una mejor lectura de los datos de inflación y desempleo en el período 1956-1975 ofrecería una curva de Phillips de pendiente positiva.

La honestidad intelectual de Friedman lo lleva a conjeturar que tal hipótesis merece nuevos estudios, pero que no podrán ser consistentes con su propio modelo teórico, basado en la neutralidad del dinero en el largo plazo.

41 Véase Friedman (1987).

42 Véase Ravier (2010b y c). 
Esta "observación de Friedman" se fortalece con los estudios de Robert Lucas. ${ }^{43}$ También fue estudiada con nueva evidencia empírica por William Niskanen. ${ }^{44}$ Yo mismo planteo en mi tesis doctoral una respuesta a su observación, pero ya abandonando el paradigma monetarista, y basándolo en la literatura de la Escuela Austríaca, donde las contribuciones de Friedrich Hayek se vuelven de especial importancia. ${ }^{45}$ Recientemente, Robert Mulligan ${ }^{46}$ toma también el trabajo de Niskanen para rehabilitar una curva de Phillips austríaca. Lo cierto es que una xurva de Phillips de pendiente positiva solo puede ser compatible con la "no neutralidad del dinero en el largo plazo", y esto invalida parte de las contribuciones de Milton Friedman. ${ }^{47}$

\section{LIBERTAD DE ELEGIR}

Independientemente de los esfuerzos que realizó Milton Friedman por caracterizar sus contribuciones con la objetividad de un científico, tanto en vida como en la actualidad, es identificado por colegas, y también por críticos, con la ideología liberal. ${ }^{48}$

Friedman creía en los mercados libres y desconfiaba del Estado. Compartía con los miembros de la Mont Pelerin Society — a la que pertenecía - ciertos principios básicos en favor de la libertad individual, la economía de mercado, la propiedad privada y el gobierno limitado. Friedman luchó, en definitiva, por la "libertad de elegir" de los individuos.

Para ser más precisos, Friedman pensaba que la propiedad privada es la base del intercambio, la justicia y el progreso en la sociedad. Defendía el laissez faire capitalista y creía en la mano invisible de Adam Smith, en el sentido de que acciones individuales, aun cuando pueden ser egoístas, maximizan tanto el bienestar individual como el de la sociedad. ${ }^{49}$ Fue crítico de Marx y de las doctrinas

43 Véase Lucas (1973).

44 Véase Niskanen (2002).

45 La pregunta esencial que nos hacemos es cuál es la misteriosa razón por la cual la tasa natural de desempleo permanecerá inalterada tras un proceso de auge, crisis y depresión. Demostramos que el ciclo económico incluye cierta destrucción de capital que sería consistente con mayor desempleo en el largo plazo, no igual.

46 Véase Mulligan (2011).

47 Véase Ravier (2010a). Véase también Ravier (2008).

48 Véase Friedman (1966 [1962]). Véase también M. y R. Friedman (1980).

$49 \mathrm{Al}$ respecto vale recordar que inició su programa televisivo con un lápiz en la mano, recordando el artículo clásico de Leonard Read, Yo, el lápiz. 
marxistas de la explotación, así como de las nociones anticapitalistas. Defendió el libre comercio, la política de libre inmigración y la globalización. Luchó por la eliminación de barreras que impiden en ciertos países el ingreso de bienes y servicios, capitales y personas. Se opuso a los controles de cambio, a los controles sobre precios, rentas y salarios, incluyendo el salario mínimo. Creía en limitar al gobierno y en tal sentido propuso la privatización, la desregulación y la desnacionalización. Se opuso al Estado de bienestar y a los privilegios especiales. Rechazó la planificación central socialista y todas las formas de totalitarismo. Reconoció que cierta desigualdad entre los habitantes del pueblo es inevitable, pero sabía que los principios de la economía de mercado podían terminar con la pobreza. Defendió la igualdad ante la ley, para el rico y para el pobre. Refutó al keynesianismo y explicó los errores de aquellos marxistas que piensan que el capitalismo es inherentemente inestable y que requiere del gobierno para estabilizar la economía. Se opuso al déficit fiscal y a los impuestos progresivos y defendió las alternativas del mercado libre sobre la educación y la salud. ${ }^{50}$ Pero, sobre todo, Friedman fue - tanto por sus trabajos teóricos como empíricos- el mayor enemigo de la inflación, a la que definía como "un impuesto sin legislación".

Y no decimos esto solo por su trabajo académico. Friedman se arremangó, viajó a muchos países y conversó con mandatarios. En algunos países incluso fue escuchado, convirtiéndose en un actor principal de su política económica. Tal fue el caso de Chile.

\section{III.1. Chile y Augusto Pinochet}

Friedman ha sido criticado y lo seguirá siendo por haber apoyado el régimen militar de Pinochet. Pero esto es un error. Friedman, al igual que Hayek, no defendió el autoritarismo, ni descreyó de la democracia limitada.

Es cierto, sin embargo, que viajó a Chile, se reunió con Pinochet, y que, a pedido de este último, ofreció una serie de reformas que se debían tomar si el país quería abandonar el proceso inflacionario y avanzar hacia una economía de mercado. También es cierto que la política económica de Pinochet fue en gran parte la que Friedman recomendara. Pero también hay que decir que tales reformas

50 Véase Friedman (1995). Este artículo ha generado una gran discordia entre libertarios. Friedman ha sido criticado por mantener con esta propuesta las manos del Estado sobre la educación. Pero él mismo se ha defendido señalando que su intención era justamente la contraria. Esta medida es importante como "transición" hacia un sistema de educación privada. Similares respuestas, veremos más adelante, elabora Friedman ante las críticas recibidas con su propuesta monetaria. 
permitieron a Chile eliminar el déficit fiscal, terminar con el proceso inflacionario, avanzar hacia una economía de mercado y con ello constituir una clase media que presionó por el fin de la dictadura y el retorno a la democracia.

Las bases económicas de esa recuperación de Chile fueron tan fuertes que incluso los gobiernos socialistas posteriores las mantuvieron en su esencia, constituyendo a Chile como un ejemplo a seguir por el resto de los países latinoamericanos que quieran alcanzar un desarrollo sustentable.

Chile presenta hoy una moneda sana y estabilidad; una economía abierta, insertada en el mundo; equilibrio fiscal y un nivel de gasto público que no llega al 20 por ciento del PIB; un sistema de pensiones privatizado que es un ejemplo para el mundo, y que le ha permitido a sus trabajadores retirados disfrutar de mejores pensiones y a la economía disfrutar de un mayor ahorro que sustenta las inversiones de mediano y largo plazo. ${ }^{51}$

Este libro reúne en su segundo ensayo los remedios de Milton Friedman a la enfermedad de la inflación. Una cura que precisamente hizo efecto en el caso de Chile, y por el cual todos debiéramos estar agradecidos.

III.2. Los remedios a la enfermedad de la inflación

Más arriba, en las proposición número 8, Milton Friedman expone lo que considera la única causa de la inflación: "la inflación es siempre y en todas partes un fenómeno monetario" en el sentido de que es y solo puede ser producida por un aumento más rápido de la cantidad de dinero que de la producción.

Friedman contrasta empíricamente dicha hipótesis sobre una serie de países. Tal es el caso de Estados Unidos, Alemania, Gran Bretaña y Japón, en cada caso para el período 1964-1973.

Sin embargo, Friedman no se limita únicamente a mostrar la evidencia, sino que se pregunta también por qué las autoridades monetarias incurren en estos excesos monetarios. $\mathrm{Y}$ encuentra tres respuestas que vale la pena mencionar. Primero, y esto es algo que viene de siglos atrás, los gobiernos, obligados a gastar,

51 Las ideas de Milton Friedman fueron la clave de la recuperación chilena. La carta que le escribiera al presidente Pinochet el 21 de abril de 1975 se encuentra online y también ha sido publicada en inglés en el libro de memorias de Milton y Rose Friedman titulado Two Lucky People, ya mencionado con anterioridad. 
no se atreven a elevar abiertamente las cargas impositivas. Por ello recurren al impuesto inflacionario que es encubierto. Segundo, porque desde Keynes en adelante, los gobiernos asumen la responsabilidad de alcanzar el pleno empleo. Esto es un fenómeno cultural que será difícil cambiar. Tercero, por las erróneas políticas monetarias que aplica la banca central, creyendo también desde Keynes que le cabe a la autoridad monetaria controlar científicamente las variables monetarias, y en particular los tipos de interés.

Pasando ahora al remedio, Friedman acierta en señalar que "la única forma de acabar con la inflación estriba en no permitir que el gasto público crezca tan rápidamente". Y es que generalmente la inflación proviene de "monetizar el déficit fiscal". Ahí es donde el problema de la inflación deja de ser un asunto técnico, para pasar a ser un problema político.

\section{III.3. La academia y la política}

Este es quizás un punto que distingue a Friedman de Ludwig von Mises, Friedrich Hayek, Murray Rothbard y otros académicos, todos miembros de la Mont Pelerin Society. Se trata de la distinción, no siempre clara, entre el rol del académico y el rol del político. El primero debe correr el eje de debate, imaginar nuevos mundos posibles, y no atender a si una propuesta de política pública es viable o no en el presente. El rol del político, o el de aquel encargado de asesorar al gobierno, es diferente. Inspirado en los debates académicos, debe atender necesariamente a aquello que la opinión pública puede digerir en el presente y trabajar a su vez para intentar que una propuesta que no es viable hoy, lo sea en el futuro. Si el primero solo atiende a lo "viable", entonces es difícil el cambio y el progreso. Si el político ignora lo "viable", su carrera no tiene futuro.

Pero Friedman tiene otra postura:

Análogamente, aquéllos de nosotros que nos hemos preocupado de la política hemos tenido que elegir no entre órdenes alternativos ideales, sino entre posibilidades alternativas realistas; realistas, me apresuro a añadir, no con respecto a su factibilidad política, que es precisamente lo que deberíamos tratar de encontrar y no eliminar de partida, sino con respecto al resultado efectivo más probable de uno y otro sistema. ${ }^{52}$

52 Véase Milton Friedman, «Un sistema monetario para una sociedad libre». Incluído en este libro. Presentado ante la Sociedad Mont Pelerin, Chile, en 1981. 
Friedman siempre tuvo un ojo en la historia económica. Aprendió por ejemplo del caso alemán, donde Ludwig Erhard suspendió controles de precios, avanzó en una reforma fiscal, terminó con la emisión de moneda, lo que no podía tener otro resultado que el "milagro alemán". Algo similar ocurrió en Japón, con el informe Dodge. Redujo el gasto público, aumentó los impuestos, eliminó el déficit, también la impresión de moneda, suspendió los controles de precios, y el resultado fue tan exitoso como el caso anterior.

Este es el modelo de transformación que él propuso para los países en problemas. Tuvo éxito en Estados Unidos o en Chile y también fue ignorado en muchos otros países. Pero nunca abandonó el esfuerzo por cambiar el mundo en el sentido que sus principios le indicaban.

\section{III.4. Un sistema monetario para una Sociedad Libre}

Como cierre, quiero hacer referencia a la crítica que Friedman recibió de algunos libertarios, en particular sobre su propuesta monetaria. Decía Rothbard, en una entrevista en la que se le consultó sobre el pensamiento de Friedman: "es excelente, excepto en moneda" 53 .

Rothbard, como tantos otros autores libertarios, han acusado a Friedman de pensar que bajo su concepción el sistema de banca central es parte de un sistema monetario ideal en una sociedad libre.

Esta confusión, proviene de analizar la propuesta monetaria más conocida en Friedman "bajo un sistema de banca central", al aplicar una regla monetaria simple, donde la cantidad de dinero aumenta en forma moderada y estable año a año, acompañando el crecimiento de la economía y manteniendo el nivel de precios estable.

Pero es aquí precisamente donde puede resultar ilustrativa la lectura de una conferencia que Friedman ofreció ante la Sociedad Mont Pelerin en 1981, en Viña del Mar. ${ }^{54}$ Allí Friedman recalcó que no existe otro aspecto importante además de la política monetaria, respecto del cual los liberales lleguen, partiendo de los mismos principios fundamentales, a conclusiones tan divergentes. Y si bien Friedman evalúa críticamente algunos de los distintos sistemas monetarios alternativos propuestos por sus colegas de la Mont Pelerin Society, no lo hace por considerarlos indeseables, sino por calificarlos de inviables.

53 Véase Ravier (2011).

54 Véase Friedman (1981). 
No se trata de volver sobre el tradicional debate entre "reglas versus discrecionalidad", sino avanzar hacia otras propuestas discutidas "entre liberales". 55

Veamos algunos extractos sobre su "crítica" a un posible retorno al patrón oro:

El retorno a un patrón oro real bien puede ser deseable, pero actualmente es imposible. Requeriría que todos los países abandonaren el uso de la política monetaria como un instrumento para afectar el empleo o los niveles de precios internos.

Y luego agrega:

¿Es probable que el resultado sea un uso extensivo del oro? Mi conjetura es que no; que ello no ocurrirá a menos que la conducta monetaria de los Estados Unidos se haga mucho peor de lo que ha sido y produzca tasas de inflación que sean un múltiplo substancial de las que hemos estado experimentando. La ventaja de un medio de intercambio común; los costos en recursos de un verdadero patrón oro; y los problemas de transición para sobreponerse al hábito y a la inercia, serán, creo yo, suficientes para impedir el surgimiento de un patrón privado realmente significativo. Sin embargo, me apresuro a agregar que éste es un juicio empírico, no una recomendación o una expresión de oposición a medidas privadas tendientes a promover el desarrollo de un patrón oro privado. Si alguno efectivamente se desarrolla, sería muy bienvenido.

Algo similar ocurre con su crítica a la propuesta de monedas competitivas de Hayek:

En resumen, en teoría hay muchas razones para aprobar las proposiciones del profesor Hayek de remover cualquier obstáculo legal al desarrollo de dinero privado competitivo. Tiene, sin embargo, poco apoyo en la experiencia esperar que surja dinero privado que se use en forma amplia en los principales países, a menos que la conducta monetaria del gobierno se haga mucho peor de lo que ha sido en el período post Segunda Guerra Mundial. Y tiene poco apoyo en la experiencia esperar tal degeneración extrema en la conducta monetaria, excepto como consecuencia de un grave conflicto militar. ${ }^{56}$

No es el lugar aquí para dar mi opinión sobre las reflexiones de Friedman a estas y otras propuestas. Pero quiero remarcar que Friedman aclaró más tarde que estaría deseoso de "abolir la Reserva Federal", y que las propuestas que plan-

55 Para ver los resultados satisfactorios de tal propuesta véase en particular su libro A program for Monetary Stability, Fordham University Press, 1959, pp. 90 ss. Para un análisis crítico véase también "Reglas versus discrecionalidad. Una ampliación del debate", en Ravier (2010): En Busca del Pleno Empleo. Estudios de Macroeconomía Austriaca y Economía Comparada, Unión Editorial, Madrid.

56 Véase también Friedman (1984). 
teara a lo largo de sus escritos, fueron siempre bajo el supuesto de que "existe la Reserva Federal". 57

"La diferencia que mantengo con personas como Murray Rothbard es que mientras yo deseo conocer cuál es mi ideal, también deseo discutir cambios que no son ideales, mientras me conduzcan en la dirección de ese ideal. Entonces aun cuando yo desearía abolir la Fed, he escrito muchas páginas sobre cómo debe manejarse la Fed, dado que existe" 58.

Y este no fue un hecho aislado. En una carta que Friedman envió a Gregory Mankiw en agosto de 2006, también señaló su voluntad de abolir la Fed:

He llegado a la conclusión de que los bancos centrales hicieron un trabajo maravilloso al tirar de la lana en los ojos de los economistas. Nos llevaron a todos a creer que el mantenimiento de un nivel de precios relativamente estable es un problema muy difícil que requiere el juicio de los más sabios de los banqueros con experiencia y gente de negocios. La facilidad con que Nueva Zelanda, Australia, Gran Bretaña, etc., han mantenido los precios relativamente estables y han reducido en gran medida la variabilidad de la inflación, sugiere que tal vez no sea un trabajo difícil en absoluto, que los ciclos del pasado no se atribuyen a la dificultad de lograr la estabilidad de precios, sino a los errores de los bancos centrales de no lograr la estabilidad de precios. Nada de lo que he observado en las últimas décadas me ha llevado a cambiar de opinión sobre la conveniencia de una regla monetaria que simplemente aumente la cantidad de dinero de mes en mes a una tasa fija. Esa regla corregiría los errores y eso es probablemente todo lo que podemos esperar obtener de un sistema monetario.

Pero aún mejor sería abolir la Reserva Federal y dar un mandato a la Tesorería para que mantenga el dinero de alto poder en un nivel constante. 59

¿Qué tan lejos se encuentra entonces la obra de Friedman de aquellos que han defendido un retorno al patrón oro, las monedas competitivas o las limitaciones constitucionales? Mi impresión es que lo que distingue a Friedman de Ludwig von Mises o Friedrich Hayek, ${ }^{60}$ o incluso de Murray Rothbard, es su interés particular por la política, por cambiar el mundo hoy, y no olvidar nunca que lo viable es lo que importa.

57 Para una mejor comprensión de la evolución del pensamiento de Milton Friedman en relación con el monopolio de la moneda, véase Selgin (2008).

58 Véase la entrevista a Milton Friedman en The Reason Magazine, "The Best of Both Worlds". 
Lamentablemente, no recuerdo el nombre de un hombre de negocios con el cual tuve el placer de conversar en la reunión anual de la Mont Pelerin Society que tuvo lugar en la Universidad Francisco Marroquín, en Guatemala, en noviembre de 2006. Tuvimos una larga conversación en la cual le comenté que, en mi opinión, la mejor propuesta para terminar con la inflación y los ciclos económicos era abolir el sistema de banca central y retornar a un sistema de banca libre, como el que alguna vez practicó Escocia o incluso Estados Unidos. ${ }^{61}$ Esta persona se definió como un gran amigo de Milton Friedman, con quien se juntaban a menudo a hablar de estas cuestiones. Y me dijo, "imagino que Milton estaría de acuerdo con esta propuesta". Prometió consultarle y escribirme con su respuesta, pero solo una semana después Milton Friedman nos abandonó para siempre.

\section{REFERENCIAS BIBLIOGRÁFICAS}

Ávila, J. (2006). Un hombre de suerte: Milton Friedman, 1912-2006. Indicadores de Coyuntura, 472, 19-21.

Bodin, J. (1569). Réponse aux paradoxes de Malestroít.

Boland, L. (1979). A Critique of Friedman's Critique. Journal of Economic Literature, 17 (2), 503-522.

Bordo, M. D., \& Rockoff, H. (2011). The Influence of Irving Fisher on Milton Friedman's Monetary Economics. NBER Working Paper No. 17267.

Cagan, P. (1965). Determinants and Effects of Changes in the Stock of Money, 1875-1960. New York: Columbia University Press. Prólogo de M. Friedman. Recuperado de http://www.nber.org/chapters/c1639.pdfhttp://www.nber.org/chapters/c1639.pdf

Caldwell, B. J. (1980). A Critique of Friedman's Methodological Instrumentalism. Southern Economic journal, 47 (2), 366-374.

Caldwell, B. J. (1984). Appraisal and Criticism in Economics: A Book of Readings. Boston: Allen and Uwin.

Cole, J. H. (1991). La obra científica de Friedman. Libertas No. 16. Recuperado de http:// www.eseade.edu.ar/wp-content/uploads/2016/08/Cole.pdf

Cole, J. (2007). Milton Friedman 1912-2006. The Independent Review: A Journal of Political Economy, 12 (1), 115-128.

Davis, W. L., Figgins, B., Hedengren, D., \& Klein, D. B., (2011). Economics Professors' Favorite Economic Thinkers, Journals, and Blogs (along with Party and Policy Views). Econ Journal Watch, 8 (2), 126-146.

De Pablo, J. C. (2011). La Escuela de Chicago en Argentina. Documentos de Trabajo UCEMA, No. 460.

Fisher, I. (1963).The Purchasing Power of Money [1911]. 2nd ed. rev. New York: Augustus, M. Kelley.

61 Véase Smith (1993 [1936]). 
Friedman, M., \& Kuznets, S. (Eds.) (1937). Studies in Income and Wealth, vol. 1. New York: National Bureau of Economic Research.

Friedman, M., \& Kuznets, S. (Eds.) (1938). Studies in Income and Wealth, vol. 2. New York: National Bureau of Economic Research.

Friedman, M., \& Kuznets, S. (Eds.) (1939). Studies in Income and Wealth, vol. 3. New York: National Bureau of Economic Research.

Friedman, M., \& Kuznets, S. (1939). Income from Independent Professional Practice, 1929-1936. NBER Bulletin, $N^{o}$ 72-73.

Friedman, M., \& Kuznets, S. (1954). Income from Independent Professional Practice. New York: National Bureau of Economic Research.

Friedman, M. (1950). Wesley C. Mitchell as an Economic Theorist. Journal of Political Economy, 58 (6), 465-493.

Friedman, M. (1967). Ensayos de Economía positiva. Madrid: Gredos.

Friedman, M. (1956). Studies in Quantity Theory of Money. Chicago: University of Chicago Press.

Friedman, M. (1957). A Theory of the Consumption Function. Princeton: Princeton University Press.

Friedman, M. (1959a). A program for Monetary Stability. New York: Fordham University Press.

Friedman, M. (1959b). The Demand for Money: Some Theoretical and Empirical Results. Journal of Political Economy, 67 (4), 327-351.

Friedman, M. (1961). The Lag in the Effect of Monetary Policy. Journal of Political Economy, 69 (5), 447-466.

Friedman, M. (1962). Capitalism and Freedom. Chicago: University of Chicago Press.

Friedman, M., \& Schwartz, A. J. (1963a). A Monetary History of the United States 18671970. Princeton: Princeton University Press.

Friedman, M., \& Schwartz, A. J. (1963b). Money and Business Cycles. Review of Economics and Statistics, 45 (1), Part 2 Supplement, 32-64.

Friedman, M. (1967). The Monetary Theory and Policy of Henry Simons. Journal of Law and Economics, 10, 1-13.

Friedman, M. (1968). The Role of Monetary Policy. American Economic Review, 58 (1), 1-17.

Friedman, M. (1970). The Counter-Revolution in Monetary Theory. Occasional Paper $N^{\circ}$ 33. Londres: Institute of Economic Affaires.

Friedman, M. (1975). Unemployment versus inflation, IEA, Lecture n. ${ }^{\circ}$ 2, Occasional paper $N^{\circ} 44$. Londres: Institute of Economic Affaires.

Friedman, M. (1977). Nobel Lecture: Inflation and Unemployment. Journal of Political Economy, 85 (3), 451-472.

Friedman, M., \& Friedman, R. (1980). Free to Choose. New York: Harcourt Brace Jovanovich.

Friedman, M. (1982). Un Sistema Monetario para una Sociedad Libre. Estudios Públicos No. 6, 165-178.

Friedman, M., \& Schwartz, A. J. (1982). Monetary Trends in the United States and the United Kingdom: Their Relation to Income, Prices and Interest Rates 1867-1975. Chicago: University of Chicago Press.

Friedman, M. (1984). Currency Competition: A Skeptical View. En P. Salin (comp.). Currency Competition and Monetary Union. (pp. 42-46). La Haya: Martinus Nijhoff,

Friedman, M. (1986). My Evolution as an Economist. En W. Breit y R. W. Spencer (comps.). 
Lives of the Laureates: Seven Nobel Economists. (pp. 77-92). Cambridge: MIT Press. Friedman, M. (1987). Rational Expectations and Inflation, por Thomas J. Sargent, Journal of Political Economy, 95 (1), 218-221.

Friedman, M. (1995). Public Schools: make them private. Briefing Papers, 23. Washington DC: Cato Institute.

Friedman, M. (2009). In Breit, W., \& Hirsch, B. T. (Eds.). Lives of the Laureates: twentythree nobel economists. (4th ed.), (pp. 67-77). Cambridge, Mass.: MIT Press.

Hayek, F. A. (1979). La inflación, el erróneo empleo del factor trabajo y el paro. En ¿Inflación o pleno empleo? México: Diana.

Huerta de Soto, J. (2002). Nuevos Estudios en Economía Política. Madrid: Unión Editorial. Hume, D. (1970). On Money, and of Interest. En E. Rotwein (Ed.). Writting in Economics. Madison: University of Wisconsin Press.

Johnson, H. G. (1984). Inflación, revolución y contrarrevolución keynesiana y monetarista, Biblioteca de Economía, 17, Buenos Aires: Hyspamerica, Ediciones Orbis.

Knight, F. H. (1935). The Ethics of Competition. London: George Allen \& Unwin.

Lucas, R. (1973). Some International Evidence on Output-Inflation Tradeoffs. The American Economic Review, 63 (3), 326-334.

Machlup, F. (1976). Essays on Hayek. New York: New York University Press.

Mulligan, R. (2011). An Austrian Rehabilitation of the Phillips Curve. The Cato Journal, 31 (1), 87-98.

Musgrave, A. (1981). Unreal Assumptions in Economic Theory: The F-twist Untwisted, Kylos, 34 (3), 377-387.

Muth, J. F. (1961). Rational expectations and the Theory of prices movements. Econometrica 29 (3), 315-335.

Nagel, E. Assumptions in Economic Theory. American Economic Review Papers and Proceedings, vol. 53 (mayo de 1963): 211-19.

Niskanen, W. A. (2002). On the Death of the Phillips Curve. The Cato Journal, 22(2), 193-98.

O’Driscoll, G. P. \& Rizzo, M. J. (2010). La Economía del Tiempo y la Ignorancia. Serie Nueva Biblioteca de la Libertad No. 40. Madrid: Unión Editorial.

Overtveldt, J. Van (2007). The Chicago school. Chicago: Agate.

Patinkin, Don (1969). The Chicago Tradition, the Quantity Theory, and Friedman. Journal of Money, Credit and Banking, 1 (1), 46-70.

Phelps, E. S. (1967). Phillips curves, expectations of inflation and optimal unemployment over time. Economica, 34 (135), 254-281.

Phelps, E. S. (1968). Money wage dynamics and labor market equilibrium. Journal of Political Economics, 76 (4), Part 2, 678-711.

Phelps, E. S. (1971). Microeconomic, foundations of employment and inflation theory. London: Macmillan.

Popper, K. (1934). The Logic of Scientific Discovery. Londres: Hutchinson.

Ravier, A. (2008). Dos tradiciones y un debate en torno a la neutralidad del dinero en el largo plazo. Revista de Análisis Institucional No. 2, 213-288.

Ravier, A. (2010a). La no neutralidad del dinero en el largo plazo. Un debate entre Chicago y Viena. Cuadernos de Economía, 29 (52), 1-19. Recuperado de http://www.scielo. 
org.co/pdf/ceco/v29n52/v29n52a01.pdf

Ravier, A. (2010b). En Busca del Pleno Empleo. Estudios de Macroeconomía Austriaca y Economía Comparada. Serie Nueva Biblioteca de la Libertad No. 41. Madrid: Unión Editorial.

Ravier, A. (2010c). La Curva de Phillips de pendiente positiva y la crisis de 2008. Procesos de mercado: revista europea de economía política, 7 (1), 71-122.

Ravier, A. (2011). La Ciencias de la Libertad. Entrevista a Murray N. Rothbard (19261995). En A. Ravier. La Escuela Austriaca desde Adentro. Historias e Ideas de sus Pensadores. Vol. 1, (pp. 85-110). Madrid: Unión Editorial.

Rosende, F. (1995). La Macroeconomía post-Lucas. Estudios Públicos No. 60. Recuperado de http://www.cepchile.cl/cep/site/artic/20160303/asocfile/20160303184056/rev60_ FRosende.pdf

Rutherford, M. (2010). Chicago economics and institutionalism. En R. B. Emmett (Ed.). The Elgar companion to the Chicago School of economics. UK: Edward Elgar.

Samuelson, P., \& Solow, R. (1960). The problem of achieving and maintaining a stable price level: Analytical Aspects of Anti-Inflation Policy. The American Economic Review, 50 (2), 177-194.

Selgin, G. (2008). Milton Friedman and the Case against Currency Monopoly. Cato Journal, 28 (2), 1-19. Recuperado de http://www.indytruth.org/library/journals/ catojournal/28/cj28n2-12.pdf

Sjaastad, L. A. (2011). Programa Cuyo: a short history. Documento de Trabajo UCEMA, No. 448.

Smith, V. C. (1993). Fundamentos de la Banca Central y de la Libertad Bancaria. Serie Nueva Biblioteca de la Libertad No. 4. Madrid: Unión Editorial y Ediciones Aosta.

Zanotti, G. J. (1996). Caminos Abiertos. Revista Libertas No. 25, ESEADE.

Zanotti, G. (2007). Intersubjectivity, Subjectivism, Social Sciences, and the Austrian School of Economics. Journal of Markets \& Morality, 10 (1), 115-141.

Zanotti, G. J. (2011). Información versus conocimiento. Madrid: Unión Editorial.

(C) 2016 por los autores; licencia otorgada a la Revista Estudios Económicos. Este artículo es de acceso abierto y distribuido bajo los términos y condiciones de una licencia Atribución-No Comercial 3.0 Unported (CC BY-NC 3.0) de Creative Commons. Para ver una copia de esta licencia, visite http://creativecommons.org/licenses/by-nc/3.0/ 